\title{
A tunable palladium nanoparticle film-based strain sensor in a Mott variable-range hopping regime
}

\author{
Bo Xie ${ }^{\mathrm{a}, *, 1}$, Peng Mao ${ }^{\mathrm{c}, \mathrm{d}, 1}$, Minrui Chen ${ }^{\mathrm{b}, 1}$, Zhaoguo Li ${ }^{\mathrm{e}}$, Chang Liu ${ }^{\mathrm{b}}$, Yuyuan Qin ${ }^{\mathrm{b}}$, Lun Yang ${ }^{\mathrm{a}}$, \\ Maocai Wei ${ }^{\mathrm{a}}$, Meifeng Liu ${ }^{\mathrm{a}}$, Xiuzhang Wang ${ }^{\mathrm{a}}$, Deyan Han ${ }^{\mathrm{f}}$, Shaozhen Li ${ }^{\mathrm{g}}$, Fengqi Song ${ }^{\mathrm{b}}$, Min Han ${ }^{\mathrm{b}}$, \\ Jun-Ming Liu ${ }^{\mathrm{b}}$, and Guanghou Wang ${ }^{\mathrm{b}}$
}
${ }^{a}$ Institute for Advanced Materials, Hubei Normal University, Huangshi 435002, P. R. China

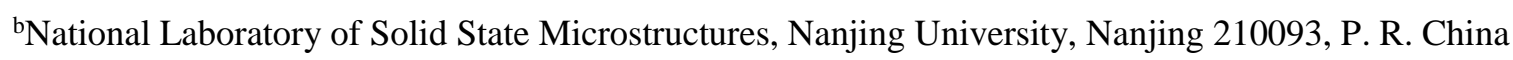
${ }^{\mathrm{c}}$ Nanoscale Physics Research Laboratory, School of Physics and Astronomy, University of Birmingham, Birmingham B15 2TT, United Kingdom
${ }^{\mathrm{d}}$ College of Electronic Science and Engineering, Nanjing University of Posts and Telecommunications, Nanjing 210023, P. R. China

${ }^{\mathrm{e}}$ Research Center of Laser Fusion, China Academy of Engineering Physics, Mianyang 621900, P. R. China

${ }^{\mathrm{f}}$ Hubei Collaborative Innovation Center for Rare Metal Chemistry, Hubei Normal University, Huangshi 435002, P. R. China

${ }^{\mathrm{g} S}$ School of Physics and Institute for Quantum Materials, Hubei Polytechnic University, Huangshi 435003, P. R. China

${ }^{*}$ Corresponding author at: Institute for Advanced Materials, Hubei Normal University, Cihu Road, No. 11, Huangshi 435002, P. R. China. Phone number: +86-18062309511.

E-mail address: xiebo@hbnu.edu.cn

${ }^{1}$ these authors contributed equally to this work

\section{Highlights}

- Palladium nanoparticle film-based strain sensor was fabricated by controllable deposition of nanoclusters onto flexible polymeric sheet in gas phase.

- Mott variable-range hopping (VRH) transport was found to be dominant in our devices.

- The gauge factor (sensitivity), which may be up to one order of magnitude greater than traditional semiconductor gauges, can be tuned by adjusting the inter-particle distance in metal nanoparticle films.

- The sensor exhibits good repeatability and reliability of their electrical responses to strains. 


\section{Abstract}

Metal nanoparticle (NP) film, an important artificial material that exhibits electronic transport properties different from bulk metals, has often been employed to fabricate micro/nano electronic devices. In this paper, Pd NP film was formed on the surface of flexible polyethylene terephthalate (PET) sheet in order to fabricate a strain sensor by means of gas-phase cluster beam deposition. Three Pd NP film-based strain sensors with different NP coverages were fabricated, and their electronic transport properties and strain sensing behaviors were investigated. Despite the differences in inter-particle coupling strength induced by different NP coverages, the Mott variable-range hopping (VRH) transport was found to be dominant in all three sensors. Sensors made of Pd NP films with lower NP coverage were found to give strain gauges with greater sensitivity, and their strain sensing calibration curves deviated from the exponential law due to variations in the gauge factor during strain loading, which was originated from the topology change of the percolation network. Sensitivity could be optimized by regulating the NP coverage, which is readily tuned during device fabrication. The tunable sensitivity, up to 1000, as well as the variety in flexible substrate materials available make them extremely promising for developing micro- and nano-sized electromechanical devices. Besides, the investigations about the reliability and hysteresis performance of the sensors were carried out. Our strain sensors show an excellent performance to the long-term bending-unbending cyclic test.

\section{Keywords}

Palladium nanoparticle film; Strain sensor; Mott variable-range hopping; Gauge factor; Inter-particle distance; Inter-particle coupling strength 


\section{Introduction}

In recent years, there has been an exponential rise in the use of fully automated equipment and the number of wearable devices [1-9], which has resulted in a great demand for stretchable strain sensors with high sensitivities and wide sensing ranges for various applications in the areas of sport, fitness tracking, electronic skins (e-skins), personal health monitoring, etc. [10-13]. Metallic foils and semiconductors have been widely used as sensing materials to fabricate commercialized strain sensors $[14,15]$. For metallic foil-based strain sensors, the typical value of the dimensionless gauge factor $\kappa \equiv \frac{(\Delta R / R)}{\varepsilon}$ (here $\varepsilon$ is the applied strain and $\Delta R / R$ is the relative change in resistance induced by the strain) is about 3, which makes for a rather low sensitivity. Semiconductor-based strain sensors usually have a rather larger gauge factor, typically about 100 , and are often preferred with respect to metallic foil-based strain sensors for small strain measurements. Despite possessing the advantage of high sensitivity, semiconductor strain sensors are fragile devices with large temperature coefficients and small working ranges ( $<5 \%$ strain). Hence, it has so far not been possible to produce metallic foil- and semiconductor-based strain sensors that can act as wearable devices and systems, in which flexibility plays a significant role, owing to the fragile and rigid nature and low sensing ranges of these devices [16].

There has been an increasing number of reports on the use of flexible substrates for strain sensor devices [7, 11-13, 16-20], a few of which explore nanoparticle (NP) thin film assemblies as sensing materials, including one report of our previous work [18]. In the case of metal NP film-based strain sensors, the metal NP monolayer could convert the mechanical strain of the substrate supporting the NP film into a reliable output signal based on inter-particle electron tunneling (see Fig. 1). The resistance of the NP film dramatically increases/decreases when tensile/compressive strain is applied. Herrmann et al. [21] used a tunneling model to describe the strain gauge behavior, which predicted the dependence of the gauge factor (sensitivity) on several parameters, in particular, the NP size, the inter-particle separation gap, and the conductance of the linking molecules. According to the theoretical model proposed by Herrmann et al., the gauge factor strongly depends on the inter-particle separation distance and the size of the NPs.

Moreover, the transport mechanisms of nanogranular systems, which are strongly dependent on the inter-particle coupling strength, were reviewed by Beloborodov et al. [22]. For this theoretical framework, the transport mechanisms of nanogranular metals can differ significantly due to the possible wide range of inter-particle coupling strengths. The electronic properties of nanogranular 
films can be easily adjusted by tuning the mean inter-particle separation, since the inter-particle tunnel coupling strength has an intrinsically exponential dependence on the inter-particle separation. In a weak-coupling regime, the temperature-dependent conductivity of the metal NP film ordinarily shows Arrhenius-type $\left(\sigma(T) \propto \exp \left(-\frac{\Delta_{M}}{k_{B} T}\right)\right)$ and/or Efros-Shklovskii variable-range hopping (ES VRH) behavior $\left(\sigma(T) \propto \exp \left[-\left(\frac{T_{0}}{T}\right)^{1 / 2}\right]\right)$, where $\Delta_{M}$ denotes the Mott gap and $T_{0}$ is a material-dependent constant, which is similar to typical semiconductor transport behavior [23-26]. In contrast, the metal NP film in a strong-coupling regime predominantly exhibits "metallic" behavior, which leads to two corrections of the diffusive conductivity [22].

In our recent work [27], another electrical transport behavior, Mott VRH, was observed in Pd NP film. Obviously, electrical transport mechanisms have a strong influence on the strain sensing behavior. In order to employ metal NP films as strain sensing materials and achieve a high gauge factor, it is necessary to investigate the correlation between charge transport mechanisms and the strain sensing gauge factor. In this work, we report on the association between the electrical transport mechanisms and the strain sensitivity of Pd NP film. Pd NP film-based strain sensors were fabricated by the gas-phase deposition of Pd nanoclusters with controlled coverage on flexible sheets of polyethylene terephthalate (PET). Pd NP films with different inter-particle separations were formed by monitoring the resistance of the Pd NP films during Pd nanocluster deposition. The Pd NP films thus fabricated displayed Mott VRH electrical transport mechanisms due to the weak coupling strength between the Pd nanoparticles. The strain sensing behaviors of the Pd NP films in the Mott VRH electrical transport regime were then investigated.

\section{Experimental methods}

\subsection{Device fabrication}

Fig. 2 is a schematic illustration of the preparation process for strain sensor devices, which was carried out at room temperature. A PET sheet of $0.5 \mathrm{~mm}$ thickness was used as the flexible substrate on which a pair of silver interdigital electrodes (IDE) with $35 \mu \mathrm{m}$ gap were deposited by mask evaporation deposition. Then Pd NP deposition was carried out using a nanocluster beam deposition system consisting of a high vacuum chamber equipped with a magnetron plasma gas aggregation cluster source $[28,29]$. The operating parameters for the cluster source can be found in our previous paper [18]. The deposition rate was precisely controlled at $0.2 \pm 0.05 \AA / s$, monitored by a quartz crystal microbalance. The cluster beam covered the whole area of the IDE. The resistance across the 
IDE gap was monitored in real time with a source meter (Keithley 2601B) by applying a bias of $1 \mathrm{~V}$ during deposition. The deposition was terminated when the predetermined resistance value, defined as the initial resistance $R_{0}$, was attained and then a Pd NP film-based strain sensor was fabricated.

\subsection{Structural characterization}

The microstructures of the fabricated Pd NPs were characterized using a field emission transmission electron microscopy (TEM, FEI TECNAI F20s TWIN). In order to perform the TEM observation, the Pd NPs were deposited on amorphous carbon films supported by copper grids that were located beside the IDE on the PET substrate, simultaneously with the fabrication of the sensor devices.

\subsection{Electrical transport measurements}

The electrical transport properties of the Pd NP films were investigated by measuring the temperature-dependent DC resistance over the temperature range 100-300 K. A closed-cycle cryostat (Janis CCS450) with a temperature controller (Cryogenic Model 32B) was used to control the temperature precisely during the experimental measurements. The current and voltage were monitored using a source meter (Keithley 2601B) with a bias of $1 \mathrm{~V}$.

\subsection{Strain sensing measurements}

In order to measure the degree of strain sensing, the strain sensors were subjected to a series of bending operations. The sensors, placed in a home-made measuring apparatus, were subjected to bending using a micrometer at room temperature, as shown in Fig. 3(a). A photographic image and corresponding schematic diagram of the measurement configuration setup for strain sensing is given in Fig. 3(b). The strain $\varepsilon$ can be expressed as follows [30]:

$$
\varepsilon=\frac{T_{\mathrm{s}}}{2 r_{\mathrm{b}}}
$$

where $T_{\mathrm{s}}$ is the substrate thickness and $r_{\mathrm{b}}$ is the radius of curvature of the sample for bending in the region where the electrodes are located, which can be calculated from the deformation according to the geometry of the measurement configuration as shown in Fig. 3(c). The resistance of the Pd NP films was monitored by a source meter (Keithley 2601B) at different applied strains.

\section{Results and discussion}

Obviously, the inter-particle distance in NP films decreases with increase in the NP coverage. Considering that smaller inter-particle distances are responsible for stronger inter-particle electronic coupling, there should be a positive correlation between the inter-particle coupling strength and the NP coverage. To investigate the effect of the strength of the tunneling coupling on the strain sensing 
behaviors of Pd NP films, films with different coverages were precisely fabricated by monitoring the resistance across the IDE gap in situ. Fig. 4 shows the evolution of the resistance of a typical Pd NP film during NP deposition. The resistance is of the order of $10^{10} \Omega$ in the initial stage of deposition. Such a large resistance indicates that there are no current pathways throughout the IDE because of the low density of NPs on the surface. As more NPs are deposited onto the surface, the inter-particle distance reduces and current pathways gradually form throughout the IDE. As a result, the resistance begins to decrease abruptly at $880 \mathrm{~s}$. Deposition was terminated at $920 \mathrm{~s}$ and a sensor sample was obtained. The resistance decreased slightly after the deposition and was finally stabilized at 9.6 M $\Omega$ after $1600 \mathrm{~s}$. In this way, three samples with initial resistance $R_{0}$ of $0.058,0.535$, and $9.615 \mathrm{M} \Omega$ (denoted as samples M1, M2, and M3, respectively) were fabricated.

The TEM micrographs of samples M1, M2, and M3 are shown in Fig. 5(a), (b), and (c), respectively, in which the Pd NPs are predominantly cuboid and form numerous closely spaced particle-assembling areas. In general, the three samples have different average inter-particle distances due to the obvious differences in NP coverage. A compact film, which can be attributed to a longer deposition time, consisting of closely spaced Pd NPs can be observed for sample M1 (Fig. 5(a)). As can be seen, most of the neighboring NPs of sample M1 contact each other. However, it can be seen from the high-resolution TEM (HRTEM) image shown in the inset to Fig. 5 that these adjacent NPs did not coalesce to form a continuous metallic film but maintained their own individual shapes and/or formed interfaces with adjacent NPs.

As shown in Fig. 5(b) and (c), samples M2 and M3 are both discontinuous films composed of isolated Pd NPs. The surface NP coverages of samples M1, M2, and M3, obtained by analyzing the TEM micrographs using ImageJ software, are about 59.34\%, 40.97\%, and 26.23\%, respectively. In all three samples, most of the Pd NPs were preferentially distributed on the substrate with a certain fractal structure rather than a uniform random distribution, and formed a large number of nanogaps. As a result, these nanogaps led to numerous quantum transport pathways based on percolation in the substrate.

The sizes of the Pd NPs were analyzed statistically, and the distributions of all three samples fitting a log-normal dependence are presented in Fig. 5(d)-(f) with probable sizes of 7.9, 8.3, and $8.2 \mathrm{~nm}$ for samples M1, M2, and M3, respectively.

Although the inter-particle distances could be obtained from the TEM images, it was difficult to directly determine whether the NP films were in weak- or strong-coupling regimes by analyzing the TEM images because of the nanostructure complexity induced by mesoscopic disorder in the NP 
films. Therefore, electron transport measurements were carried out in order to investigate the electrical transport behaviors of our devices with different NP coverages.

Fig. 6 shows the plots of resistance $R$ of samples M1, M2, and M3 in the temperature range 100 to $300 \mathrm{~K}$. The resistance of the three samples increases monotonically with decreasing temperature $T$ over the entire temperature range, indicating that all of the $\mathrm{Pd} \mathrm{NP}$ films exhibited semiconductor-like resistive behavior with a negative temperature coefficient. Plotting $R$ (log scale) versus $T^{-1 / 4}$, the $\lg R-T^{-1 / 4}$ curve shows agreement with a linear curve fit. This temperature dependence of resistance is well described by the Mott VRH mechanism [31]:

$$
R=R_{\mathrm{M}} \exp \left[\left(T_{\mathrm{M}} / T\right)^{1 / 4}\right]
$$

where $R_{\mathrm{M}}$ is the resistance parameter and $T_{\mathrm{M}}$ is the Mott characteristic temperature whose value depends on the electronic density of states at the Fermi level $N\left(E_{\mathrm{F}}\right)$ and the localization length $\xi[31$, 32]:

$$
T_{\mathrm{M}}=\frac{18}{k_{\mathrm{B}} N\left(E_{\mathrm{F}}\right) \xi^{3}}
$$

In Fig. 6, the slopes of the linear $\lg R-T^{-1 / 4}$ curves give values of the Mott characteristic temperature $T_{\mathrm{M}}$ of $4.12 \times 10^{3}, 6.47 \times 10^{3}$, and $2.03 \times 10^{4} \mathrm{~K}$ for samples M1, M2, and M3, respectively. Because Mott VRH electrical transport behavior was observed in all three samples, it can be concluded that these samples are in a weak-coupling regime.

Fig. 7(a) displays the measured relative resistance variation $\Delta R / R_{0}$ as a function of applied strain $\varepsilon$ for the three samples, which can be used as a sensing calibration curve for the sensors. At first glance, these data suggest an exponential correlation, which is consistent with previous studies [18, 21, 33]. As previously reported, a simplified model was proposed to describe the quantitative relationship between relative resistance variation $\Delta R / R_{0}$ and strain $\varepsilon$ [21]:

$$
\Delta R / R_{0}=\exp (\kappa \varepsilon)-1
$$

where the parameter $\kappa$, defined as the gauge factor, characterizes the sensitivity of the strain sensors. According to this model, the exponential law

$$
y=y_{0}+A \exp \left(R_{0} x\right)
$$

was used to fit the data of Fig. 7(a), where the parameters $y_{0}$ and $A$ are fixed with values of -1 and 1 , respectively. From the fitting result (Fig. 7(c)-(e)), it is interesting to note that the relative changes in conductance of sample M1 can be fitted very well by Eq. (5), while the fitting function becomes invalid for the M2 and M3 samples data. It can therefore be concluded that Eq. (4) is suitable for 
modeling the strain-sensing behavior of our devices with shorter rather than longer inter-particle distances.

Regarding the physical model proposed by Herrmann et al. [21], the gauge factor $\kappa$ was viewed as a constant in Eq. (4). In practice, we incline to the view that the gauge factor varies with the inter-particle distance. As a result, the gauge factor should be considered as a function of strain because of the correlation between strain and inter-particle distance. As tensile strain was applied to the samples by bending the PET substrate, the length of the substrate varied from $L_{0}$ to $L=\Delta L+L_{0}$, inducing a strain $\varepsilon=\Delta L / L_{0}$, and the average inter-particle distance increased from $l_{0}$ to $l=\Delta l+l_{0}$. Assuming that the NPs can be viewed as rigid points, the relationship $\varepsilon=\Delta L / L_{0} \cong \Delta l / l_{0}$ is satisfied. Moreover, a dimensionless conductance parameter $g=G /\left(2 e^{2} / \hbar\right)$ was proposed by Beloborodov et al. [22] as an important physical parameter characterizing the inter-particle coupling strength to describe the transport properties induced by the inter-particle tunnel coupling, where $G$ is the average tunneling conductance between neighboring NPs. Denoting the average tunneling resistance between neighboring NPs as $R_{l}=1 / G, g$ can be expressed as

$$
g=\hbar / 2 e^{2} R_{l}
$$

Huth [34] investigated theoretically the evaluation of the intrinsic strain dependence of the electrical conductivity of granular metals and analyzed the relationship between the gauge factor and the inter-particle coupling strength, and found that the gauge factor $\kappa$ is proportional to the value of $-\ln g$ for the metal nanocrystal films in the VRH regime:

$$
\kappa \propto-\ln g
$$

In addition, the average tunneling resistance $R_{l}$ between neighboring NPs depends approximately exponentially on the separation gap $l$ between the NPs [35]:

$$
R_{l} \propto \exp (\beta l)
$$

where $\beta$ is a size- and temperature-dependent electron coupling term. From Eqs. (6)-(8), we can obtain

$$
\Delta \kappa=\kappa-\kappa_{0} \propto \beta \Delta l=\beta l_{0} \varepsilon
$$

where $\kappa_{0}$ is the gauge factor at $\varepsilon \rightarrow 0$. The values of $\kappa_{0}$ for samples M1, M2, and M3, which could be estimated using a linear fit to small applied strains, as shown in the insets of Fig. 7(c)-(e), were $1.417,36.93$, and 50.96, respectively. The above result indicates that the initial average 
inter-particle distance $l_{0}$ without strain has an impact on the variation in gauge factor $\Delta \kappa$ when stress is applied to the devices.

According to the International Union of Pure and Applied Chemistry (IUPAC) definition, "sensitivity" is the slope of the calibration curve, that is, the sensor response versus strain $\kappa=\frac{d\left(\Delta R / R_{0}\right)}{d \varepsilon}$. We plotted the gauge factor, which was extracted directly from the derivative of the sensing calibration curves of Fig. 7(a), as a function of the strain in Fig. 7(b). It is clear that the approximate relationship between gauge factor and strain is linear, which is in agreement with Eq. (9). As quantitatively analyzed in Fig. 7(b), the slopes of the three fitting curves, which represent the multiplication effect of the initial average inter-particle distance on the variation in gauge factor during strain loading, are $0.74,11.59$, and $51.79 \%^{-1}$ for samples M1, M2, and M3, respectively, indicating that a larger initial average inter-particle distance is responsible for a larger variation in the gauge factor.

In the case of the NP film with high NP coverage corresponding to low initial resistance $R_{0}$, the value of the initial average inter-particle distance $l_{0}$ is so small that a relatively small variation in the gauge factor is produced. Namely, the gauge factor of the sample with high NP coverage can be viewed as a constant. In contrast, the samples with higher initial resistance values tend to have greater initial average inter-particle distances, which results in a significantly larger variation in the gauge factor when stress is applied to the devices, suggesting that the gauge factor cannot be viewed as a constant, but as a variable depending on the strain. On the basis of this analysis, the deviation in the strain sensing calibration curves of our devices from the theory proposed by Herrmann et al. can be explained by taking into account the variation in the gauge factor during strain loading.

However, it is much easier and deeper to understand the deviation by considering the morphology changes of the NP films with different coverage during strain sensing. We note that the NP assemblies are not monolayer-structure but three-dimensional (3D) stacking morphology with increased NP coverage from the characterization results as shown in Fig. 5. In these samples with lower NP coverages (M2 and M3), featuring monolayer-structure, the strain had an important influence not only on the inter-particle gaps but also the percolation network topology. The topology changes of the percolation network offer additional resistance variation, leading to a more significant increase of the gauge factor. In the case of sample M1, the NPs in upper layer of the 3D stacking morphology form bridges between NPs in the lower layer, so the percolation network topology would remain homogeneous over strain, resulting in a relatively small overall variation of resistance and gauge factor [33]. Ultimately, the essential reason of the deviations of the strain 
sensing calibration curves from the exponential law is the topology change of the percolation network during strain loading for the NP film-based strain sensors with different NP coverages.

On the basis of the above experimental results and analysis, we can confirm that the gauge factor is strongly influenced by the inter-particle distance, which is controllable in the fabrication process by varying the NP coverage. Compared to metallic foils and semiconductor-based strain sensors that have a fixed gauge factor, the sensitivity of metal NP film-based strain sensors is tunable by varying the inter-particle distance. It was also found that with increase in the strain, the gauge factor of our devices rose linearly, up to a value of 1000 (Fig. 7(b)), which is one order of magnitude greater than the gauge factor of semiconductor gauges (typically about 100). Another advantage of our devices, besides the high sensitivity, is that this metal NP film can be made suitable for many applications by choosing materials with suitable mechanical properties as the substrate. For example, performing the deposition of metal NPs on materials with good elasticity, such as polydimethylsiloxane (PDMS), will enable the devices to overcome the limitation of the low dynamic range of both metallic foil- and semiconductor-based strain sensors. Gauge damage can be also avoided owing to the discontinuous morphology of films based on discrete NPs. In addition, it can also be noted from Fig. 6 that the Pd NP film with a lower initial resistance has a higher Mott characteristic temperature, indicating that the resistance varies with temperature more significantly. This means that the strain sensing response may be influenced by the variation in temperature, which also induces a considerable change in conductance. If Pd NP films, especially those with low NP coverage, are used as strain sensors, a way of carefully compensating the temperature is critically required. Moreover, although an NP film with higher initial resistance provides much higher sensitivity (see experimental data in Fig. 7) and results in lower power consumption, the signal-to-noise ratio will degrade with the increasing resistance of devices. Therefore, to improve the performance of metal NP film-based strain sensors, a low initial resistance is necessary. However, many different factors, such as substrate materials and signal-to-noise ratio, must be considered.

On the other hand, flexible electronic is an emerging field which requires sensing materials with stable and robust electromechanical properties. Therefore, to evaluated the reliability and durability of the sensor, the sample with initial resistance $R_{0}$ of $0.866 \mathrm{M} \Omega$ was fabricated, and a series of bending-unbending recycle tests were then performed. For estimating the electromechanical performance of the NPs-based strain sensor under bending-unbending cycles, the both ends of the sample were clamped by two motor-controlled sliders (see Fig. 8(a)). The sensor was subjected to 
bending-unbending cycles when the two sliders, driven by a stepper motor, move back and forth. The moving distance of the slider in bending state was termed as $\Delta d$ (see the inset of Fig.8(c)), which is positively correlated with the strain of the device in bending state. Fig. 8(b) illustrates the relative resistance variation of the sensor responding to a series of stair-type bending cycles produced from programmed steps. According to the experimental data profile of $\Delta R / R_{0}$ versus $\Delta d$, as shown in Fig. 8(c), it is found that the relative resistance variation exhibits a stair-type change, and presents a loop as the $\Delta d$ increases and decreases gradually. Moreover, an approximately linear relationship between $\Delta R / R_{0}$ and $\Delta d$ in bending steps ( $\Delta d$ increasing) is observed. In bending-release steps ( $\Delta d$ decreasing), $\Delta R / R_{0}$ and $\Delta d$ are not linear relation on the contrary, indicating the existence of hysteresis in response to the bending and bending-release processes. However, $\Delta R / R_{0}$ still fall back close to zero with a negligible baseline drift (less than $0.22 \%$ after $6 \Delta d$ increasing and decreasing cycles) when the slider moves back to the initial position $(\Delta d=0)$, showing the outstanding reliability of our NPs-structured strain sensors.

To further investigate the repeatability, hysteresis, and baseline drift of our device, the stepper motor was programmed to drive the slider reciprocating motions for about 20.5 hours with $1.6 \mathrm{~mm}$ moving distance, corresponding to more than 1400 bending-unbending cycles. In this case, the sensor exhibits a reproducible and stable response with about $15 \%$ relative change in resistance during the whole cycling test (top image in Fig. 8(d)), confirming its good consistency and durability for practical applications, owing to high adhesive force between the Pd NPs and PET layers [36]. In addition, a very minor baseline drift (about 1.6\%) was also caused by this long-term cycling test.

Taking a considerable insight into the response curve at the initial, middle, and ending stages of the long-term test, respectively, $\Delta R / R_{0}$ increases dramatically upon bending and follows a slow gradual rise, as shown in bottom image of Fig. 8(d). Similar electromechanical characteristic that $\Delta R / R_{0}$ gradually recovered to the baseline can be -observed at the bottom of the curve when the bending is released. Generally, this temporal hysteresis behavior is mainly caused by the viscoelastic nature of polymers, as well as the interaction between NPs and polymeric substrates [37]. As the experimental result show in the insets of Fig. 8(d), the gradual recovery of $\Delta R / R_{0}$ values at the initial, middle, and ending stages of the long-term test is $0.5,0.25$, and $0.16 \%$, respectively. Therefore, as testing cycles go on, the impact of hysteresis on response of the strain sensor shows gradual reduction due to the tensile stress-slackening of the viscoelastic polymeric substrates [38]. These results reveal that the NPs-based strain sensor exhibits high stability, durability and repeatability to 
In summary, Pd NP films were fabricated as strain sensors by depositing nanoclusters on PET sheets. The Pd NP film-based strain sensors with different NP coverages were obtained by monitoring the resistance of the Pd NP films during deposition. Mott VRH transport was dominant in Pd NP films. It was observed that the strain sensors with lower NP coverage, which had larger inter-particle distances and lower initial resistance, exhibited higher sensitivity and higher deviations of the strain sensing calibration curves from the exponential law, which can be attributed to the variation in originated from the topology change of the percolation network due to the morphologies of strain gauges with different NP coverages. The results showed that the gauge factor was found to be affected by the strain more strongly in the strain sensor with lower NP coverage than in those with higher NP coverage. As a consequence, the sensitivity, which can reach up to one order of magnitude greater than traditional semiconductor gauges, is tunable by varying the inter-particle distance during device fabrication. Furthermore, our sensors exhibit good repeatability and reliability of their electrical responses during the long-term bending-unbending test with negligible baseline drift. A decreased hysteresis in their strain responses under cyclic strain loading was also observed. Since a large number of materials with diverse mechanical behaviors can be used as substrates for our devices, such tunable strain sensors offer new opportunities for developing microand nano-sized electromechanical devices, including as electronic skin (e-skin), micropressure transducers, and nano-level vibration sensors.

\section{ACKNOWLEDGMENTS}

The work was supported by the National Natural Science Foundation of China (Grants 61301015, 11604161, 11604310, and 11627806), the Training Program of the Major Research Plan of the National Natural Science Foundation of China (Grant 91622115), European Union's Horizon 2020 research and innovation programme under the Marie Skłodowska-Curie Grant (Grant 752102), the Natural Science Foundation of Jiangsu Province (Grant BK20160914), and the Promotion Program of Hubei Normal University. 
[1] I. Colomina, P. Molina, Unmanned aerial systems for photogrammetry and remote sensing: A review, Isprs Journal of Photogrammetry and Remote Sensing, 92(2014) 79-97.

[2] C. Zhang, J.M. Kovacs, The application of small unmanned aerial systems for precision agriculture: a review, Precision Agriculture, 13(2012) 693-712.

[3] R.W. Beard, T.W. McLain, M.A. Goodrich, E.P. Anderson, Coordinated target assignment and intercept for unmanned air vehicles, Ieee Transactions on Robotics and Automation, 18(2002) 911-22.

[4] M. Amjadi, K.-U. Kyung, I. Park, M. Sitti, Stretchable, Skin-Mountable, and Wearable Strain Sensors and Their Potential Applications: A Review, Adv Funct Mater, 26(2016) 1678-98.

[5] J. Park, I. You, S. Shin, U. Jeong, Material Approaches to Stretchable Strain Sensors, Chemphyschem, 16(2015) 1155-63.

[6] S. Zhao, R. Zhu, Electronic Skin with Multifunction Sensors Based on Thermosensation, Adv Mater, 29(2017)

[8] A. Miyamoto, S. Lee, N.F. Cooray, S. Lee, M. Mori, N. Matsuhisa, et al., Inflammation-free, gas-permeable, lightweight, stretchable on-skin electronics with nanomeshes, Nat Nanotechnol, 12(2017) 907-13.

[9] A. Chortos, J. Liu, Z. Bao, Pursuing prosthetic electronic skin, Nat Mater, 15(2016) 937-50.

[10] X.G. Yu, Y.Q. Li, W.B. Zhu, P. Huang, T.T. Wang, N. Hu, et al., A wearable strain sensor based on a carbonized nano-sponge/silicone composite for human motion detection, Nanoscale, 9(2017) 6680-5.

[11] H. Park, D.S. Kim, S.Y. Hong, C. Kim, J.Y. Yun, S.Y. Oh, et al., A skin-integrated transparent and stretchable strain sensor with interactive color-changing electrochromic displays, Nanoscale, (2017).

[12] L. Yi, W. Jiao, K. Wu, L. Qian, X. Yu, Q. Xia, et al., Nanoparticle monolayer-based flexible strain gauge with ultrafast dynamic response for acoustic vibration detection, Nano Res, 8(2015) 2978-87.

[13] J. Lee, S. Kim, J. Lee, D. Yang, B.C. Park, S. Ryu, et al., A stretchable strain sensor based on a metal nanoparticle thin film for human motion detection, Nanoscale, 6(2014) 11932-9.

[14] J. Engel, J. Chen, C. Liu, Strain sensitivity enhancement of thin metal film strain gauges on polymer microscale structures, Appl Phys Lett, 89(2006) 221907.

[15] Kovacs, T.A. Gregory, Micromachined transducers sourcebook: New York: McGraw-Hill; 1998.

[16] A. Bessonov, M. Kirikova, S. Haque, I. Gartseev, M.J.A. Bailey, Highly reproducible printable graphite strain gauges for flexible devices, Sensors and Actuators A: Physical, 206(2014) 75-80.

[17] X. Liao, Q. Liao, Z. Zhang, X. Yan, Q. Liang, Q. Wang, et al., A Highly Stretchable ZnO@Fiber-Based Multifunctional Nanosensor for Strain/Temperature/UV Detection, Adv Funct Mater, 26(2016) 3074-81.

[18] M. Zheng, W. Li, M. Xu, N. Xu, P. Chen, M. Han, et al., Strain sensors based on chromium nanoparticle arrays, Nanoscale, 6(2014) 3930-3.

[19] D. Zaharie-Butucel, L. Digianantonio, C. Leordean, L. Ressier, S. Astilean, C. Farcau, Flexible transparent sensors from reduced graphene oxide micro-stripes fabricated by convective self-assembly, Carbon, 113(2017) 361-70.

[20] M. Segev-Bar, H. Haick, Flexible Sensors Based on Nanoparticles, ACS Nano, 7(2013) 8366-78.

[21] J. Herrmann, K.H. Müller, T. Reda, G.R. Baxter, B. Raguse, G.J.J.B. de Groot, et al., Nanoparticle films as sensitive strain gauges, Appl Phys Lett, 91(2007) 183105.

[22] I.S. Beloborodov, A.V. Lopatin, V.M. Vinokur, K.B. Efetov, Granular electronic systems, Reviews of Modern Physics, 79(2007) 469-518.

[23] Z. Li, L. Peng, J. Zhang, J. Li, Y. Zeng, Y. Luo, et al., Transition between Efros-Shklovskii and Mott variable-range hopping conduction in polycrystalline germanium thin films, Semicond Sci Tech, 32(2017).

[24] A.L. Efros, B.I. Shklovskii, Coulomb gap and low temperature conductivity of disordered systems, Journal of Physics C: Solid State Physics, 8(1975) L49.

[25] H. Fritzsche, Electrical Properties of Germanium Semiconductors at Low Temperatures, Physical Review, 99(1955) 406-19. 
[26] X. Leng, J. Pereiro, J. Strle, G. Dubuis, A.T. Bollinger, A. Gozar, et al., Insulator to metal transition in WO3 induced by electrolyte gating, npj Quantum Materials, 2(2017) 35.

[27] M. Chen, P. Mao, Y. Qin, J. Wang, B. Xie, X. Wang, et al., Response Characteristics of Hydrogen Sensors Based on PMMA-Membrane-Coated Palladium Nanoparticle Films, ACS applied materials \& interfaces, 9(2017) 27193-201.

[28] M. Han, C.H. Xu, D. Zhu, L. Yang, J.L. Zhang, Y.P. Chen, et al., Controllable synthesis of two-dimensional metal nanoparticle arrays with oriented size and number density gradients, Adv Mater, 19(2007) 2979-83.

[29] K. Wegner, P. Piseri, H.V. Tafreshi, P. Milani, Cluster beam deposition: a tool for nanoscale science and technology, Journal of Physics D: Applied Physics, 39(2006) R439-R59.

[30] J. Yin, P. Hu, J. Luo, L. Wang, M.F. Cohen, C.J. Zhong, Molecularly mediated thin film assembly of nanoparticles on flexible devices: electrical conductivity versus device strains in different gas/vapor environment, ACS Nano, 5(2011) 6516-26.

[31] N.F. Mott, Conduction in glasses containing transition metal ions, Journal of Non-Crystalline Solids, 1(1968) 1-17.

[33] C. Farcau, H. Moreira, B. Viallet, J. Grisolia, D. Ciuculescu-Pradines, C. Amiens, et al., Monolayered Wires of Gold Colloidal Nanoparticles for High-Sensitivity Strain Sensing, The Journal of Physical Chemistry C, 115(2011) $426 \quad 14494-9$.

[34] M. Huth, Granular metals: From electronic correlations to strain-sensing applications, J Appl Phys, 107(2010).

[35] K.H. Muller, J. Herrmann, B. Raguse, G. Baxter, T. Reda, Percolation model for electron conduction in films of metal nanoparticles linked by organic molecules, Phys Rev B, 66(2002) 075417-24.

430 [36] P. Jensen, Growth of nanostructures by cluster deposition: Experiments and simple models, Reviews of Modern 431 Physics, 71(1999) 1695-735.

432 [37] M. Amjadi, A. Pichitpajongkit, S. Lee, S. Ryu, I. Park, Highly Stretchable and Sensitive Strain Sensor Based on 433 Silver Nanowire-Elastomer Nanocomposite, ACS Nano, 8(2014) 5154-63.

434 [38] Y.A. Samad, Y. Li, S.M. Alhassan, K. Liao, Novel Graphene Foam Composite with Adjustable Sensitivity for 

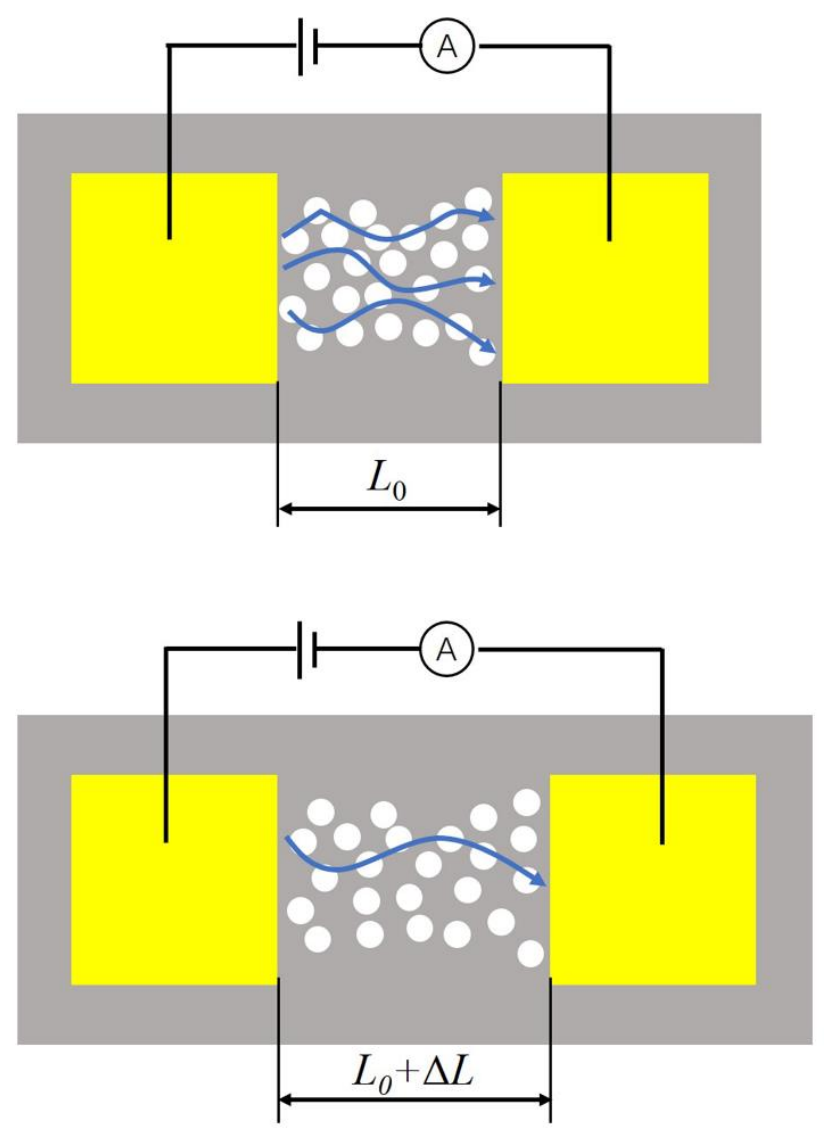

Fig. 1. Schematic depiction of the inter-particle electron tunneling in metal NP monolayer. Electrons tunnel through several NPs and form numerous current pathways (the blue arrow lines) throughout the IDE. When the substrate is stretched from $L_{0}$ to $L_{0}+\Delta L$, the inter-particle distance increases and the tunneling probability decreases, resulting in decreased current pathways and an increased resistance. 


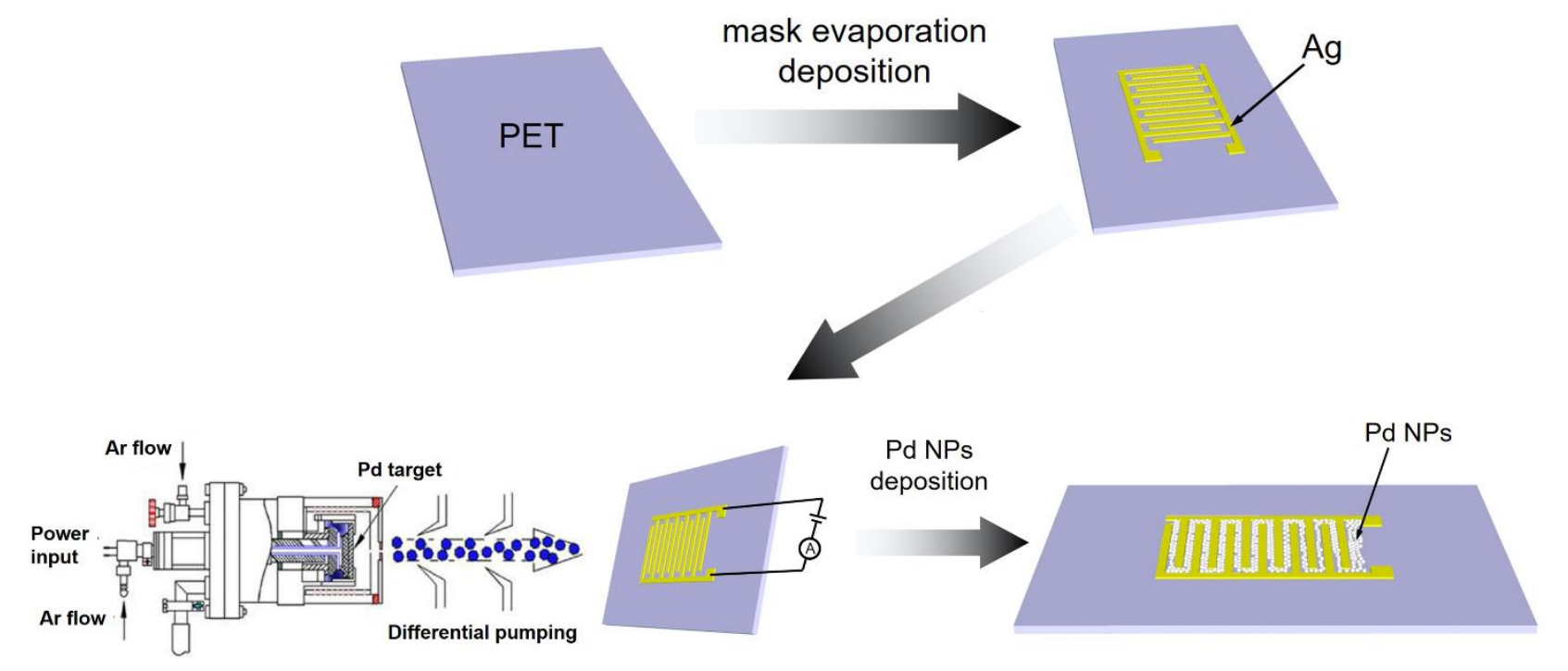

Fig. 2. Schematic illustration of the procedure used to fabricate the strain sensor based on Pd NP film. 
(a)

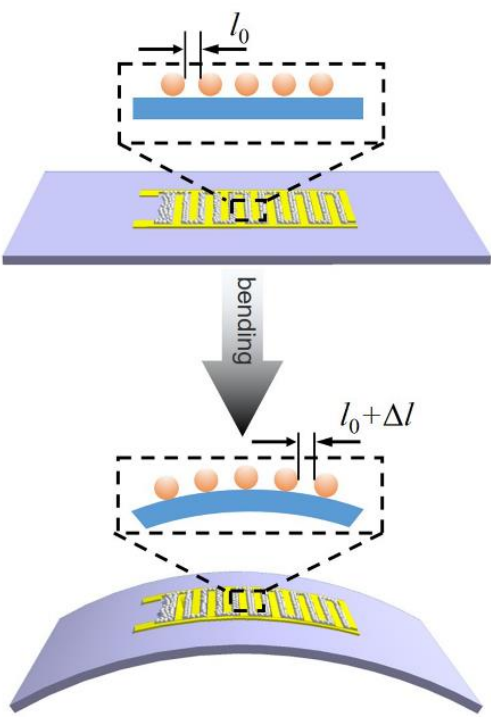

(b)

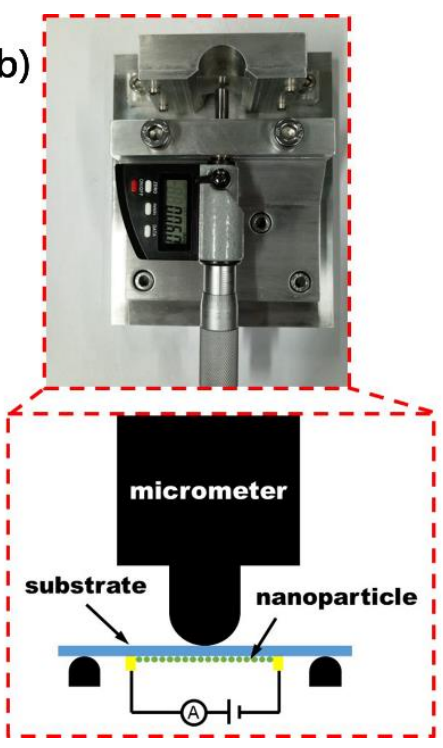

(c)

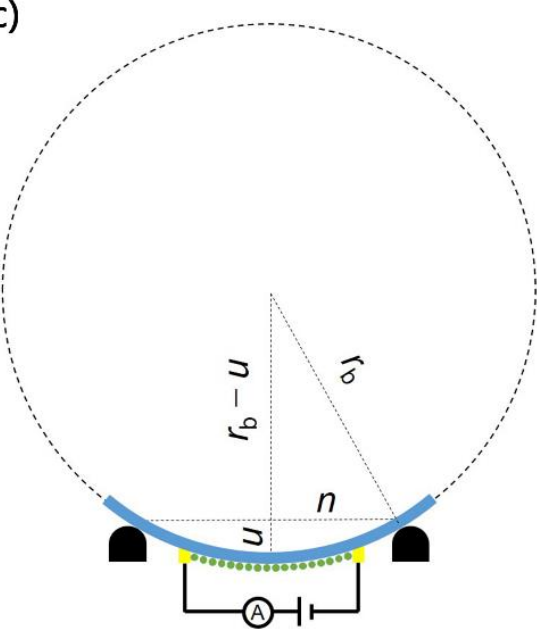

Fig. 3. Schematic diagrams of strain sensing measurements. (a) Schematic of sample straining. The inter-particle distance varied from $l_{0}$ to $l_{0}+\Delta l$ as the sensor responded to a tensile strain. (b) Measurement configuration setup for strain sensing, including a photo of the home-made measuring apparatus and a schematic diagram of the strain sensing test. (c) Geometrical relationship used to calculate the strain. The dash-dotted circle corresponds to the radius of curvature of the sample for bending. The value of $\boldsymbol{n}$ is half of the chord length, which can be obtained by measuring the distance between the two fulcrums supporting the sensor. The variable $u$ represents the distance that the micrometer travels during strain loading. 


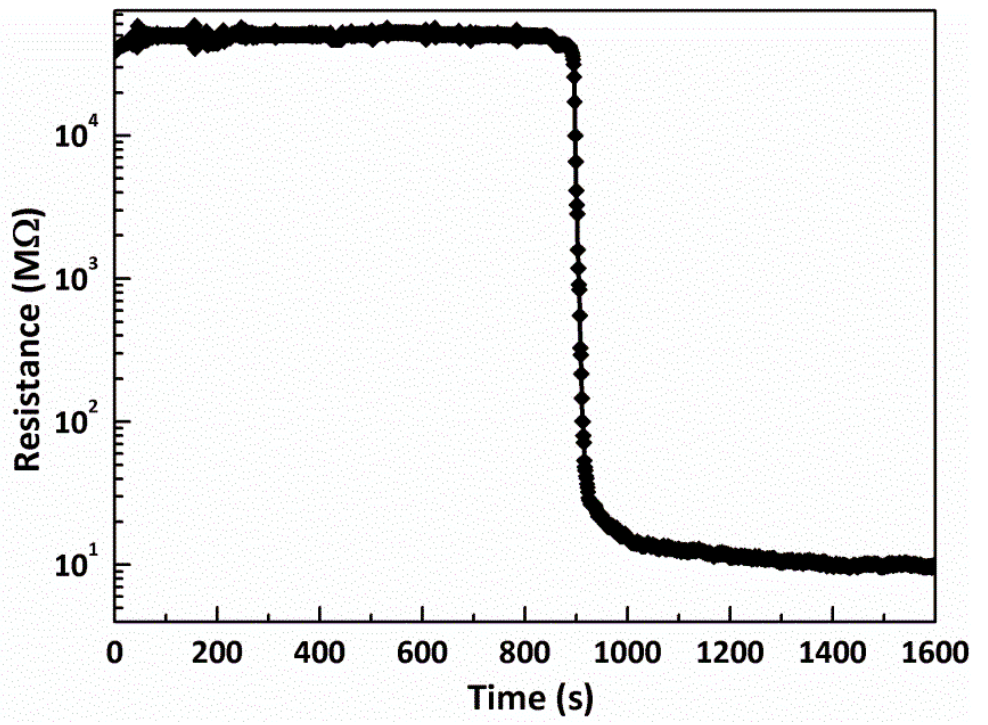

Fig. 4. Evolution of the resistance of a typical Pd NP film during Pd NP deposition. 

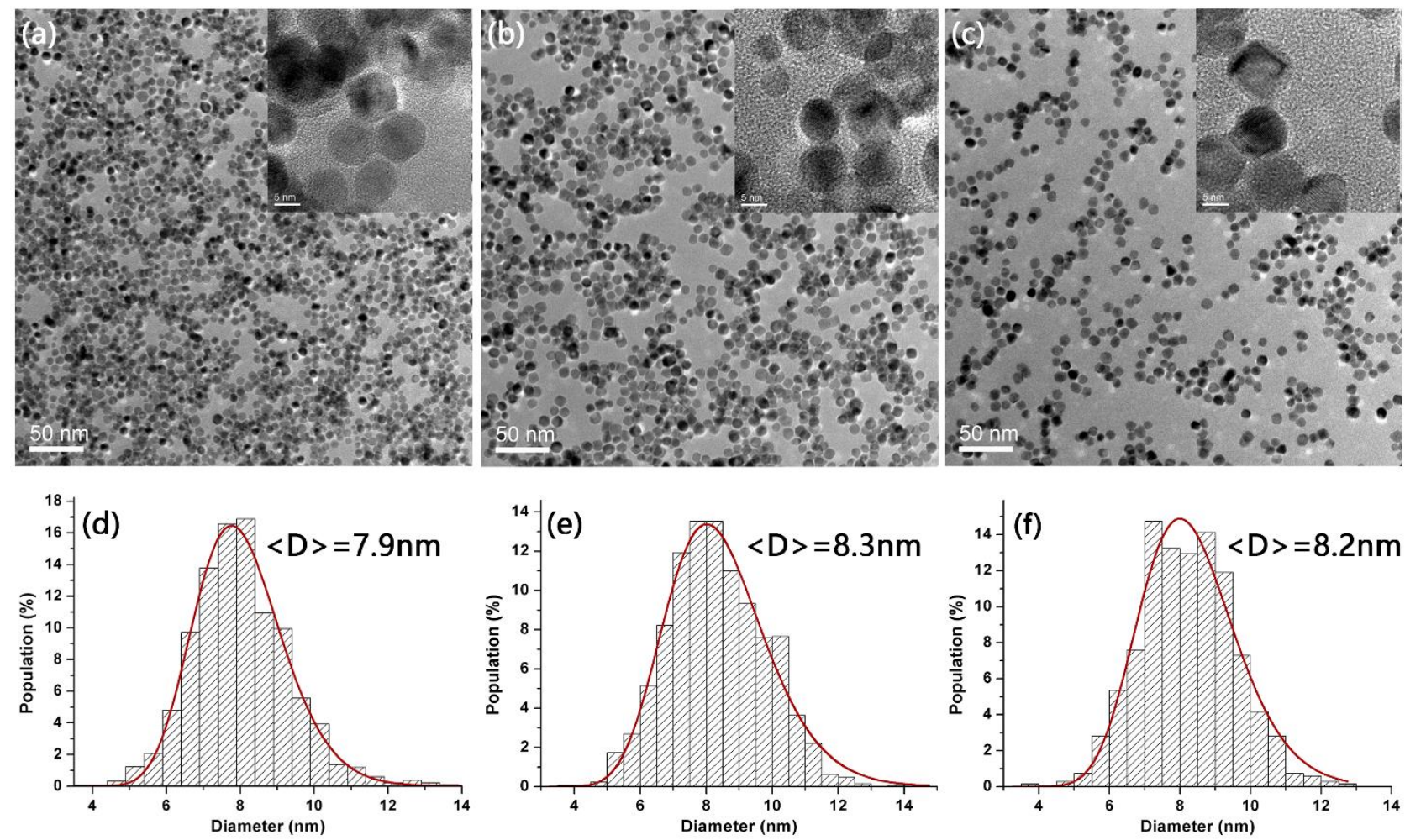

Fig. 5. The TEM images (upper row) and associated size distributions (lower row) of Pd NP film-based strain gauges with three different NP coverages: (a) and (d) M1; (b) and (e) M2; (c) and (f) M3. The insets are HRTEM images showing details about the morphology of a few neighboring NPs in each samples. The mean diameters of the NPs are marked on the histograms of the three size distributions. 

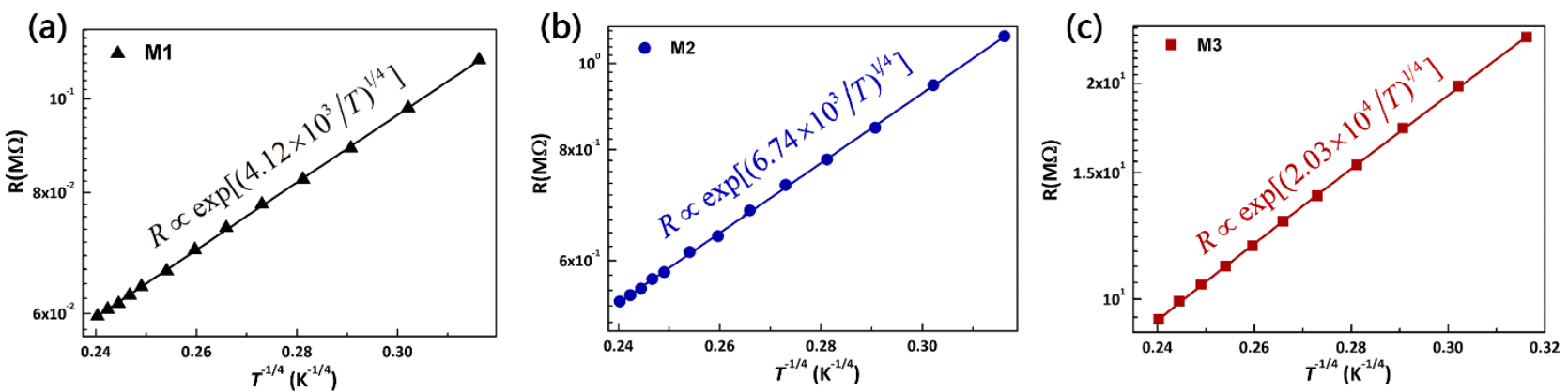

Fig. 6. Resistance of samples M1, M2, and M3 plotted against $T^{-1 / 4}$. The solid curves fit the experimental data with $R \propto \exp \left[\left(T_{M} / T\right)^{1 / 4}\right]$. 

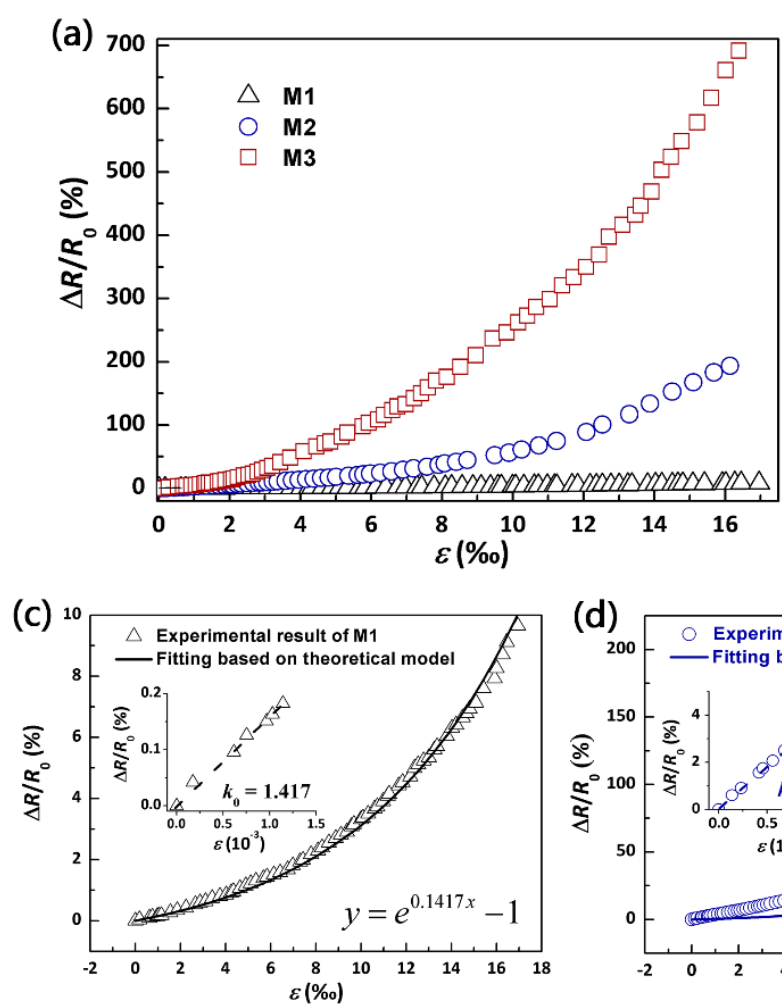

(b)
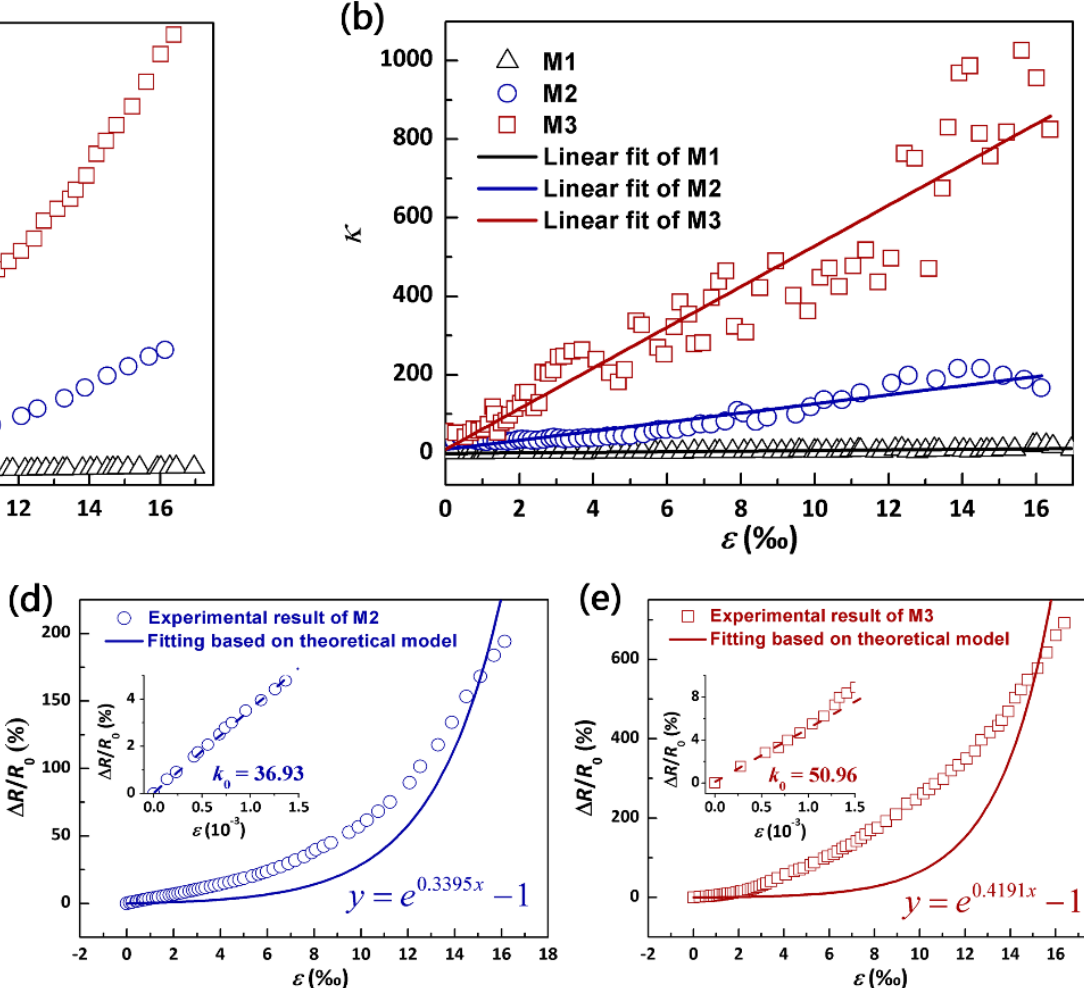

Fig. 7. (a) Relative resistance variation and (b) gauge factor as a function of the applied strain for samples M1, M2, and M3. The solid lines are the linear fit, and their corresponding fitting functions are shown in the lower-right corner. (c)-(e) Fitting results of strain sensing calibration curves shown in (a) according to the exponential law $y=\exp \left(R_{0} x\right)-1$. These insets provide a magnified view of the low-strain region, in which the gauge factor $\kappa_{0}$ at $\varepsilon \rightarrow 0$ can be obtained from the slope of the dashed line. 
(a)

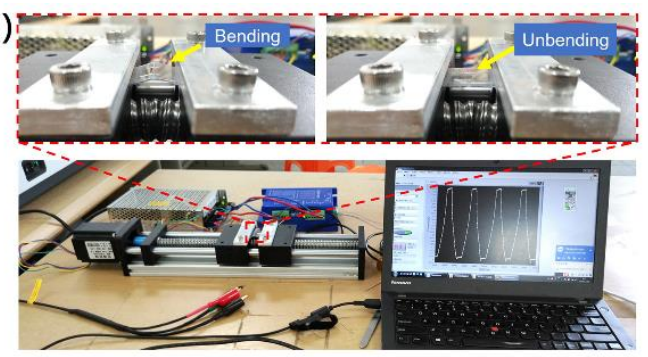

(b)

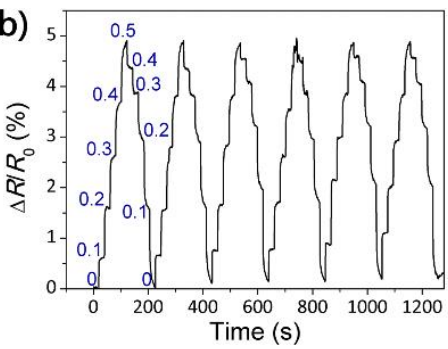

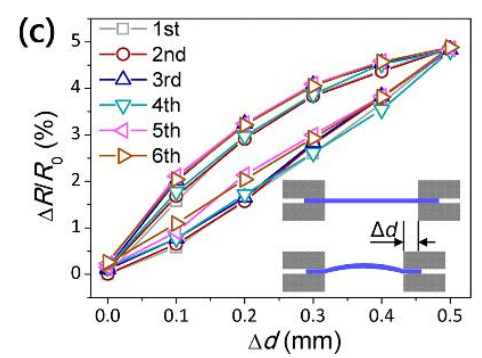

(d)

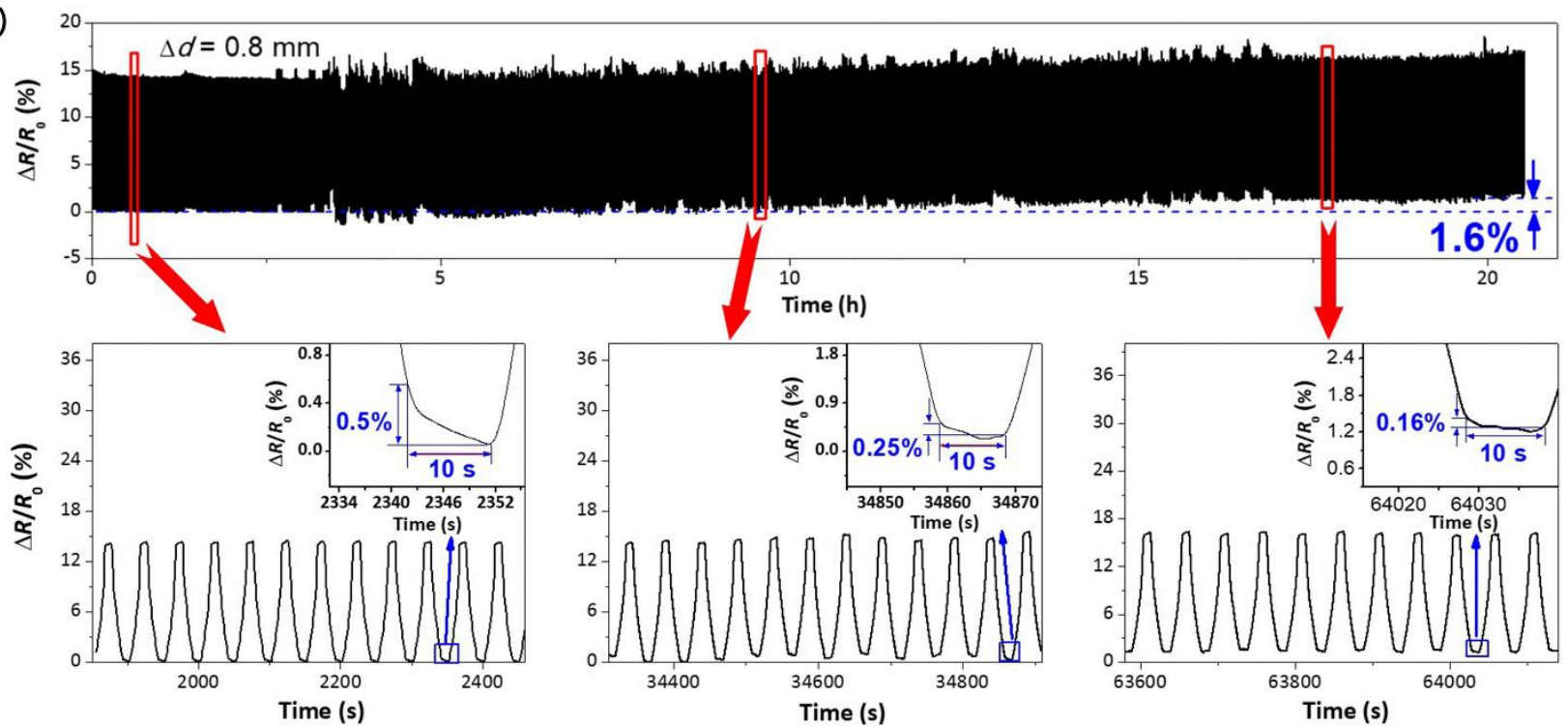

Figure 8. Electromechanical response of strain sensor based on Pd NP film. (a) Photograph of the test equipment. The details of the sensor clamped by two sliders under bending and unbending are illustrated in the enlarged views, respectively. (b) Relative resistance variation $\Delta R / R_{0}$ plotted against time when one of the two sliders move towards another step by step. The moving distance $\Delta d$ (in $\mathbf{m m}$ ) of the slider for each strain loading is marked at each response step. (c) Relative resistance variation $\Delta R / R_{0}$ of the sensor calculated from part (b) as a function of the moving distance $\Delta d$. Inset shows the illustration of the sensor was bended from unbending state due to the slider moving distance $\Delta d$. (d) Performance of the sensor under more than 1400 bending-unbending cycles with a slider moving distance $\Delta d$ from 0 to $1.6 \mathrm{~mm}$. The three bottom figures show some typical cycles at the initial, middle, and ending stages of the testing process, respectively. The insets in each bottom figure show the details about the gradual release of the $\Delta R / R_{0}$ values. Each bending and unbending state was hold $10 \mathrm{~s}$. 